\title{
The significance of coagulation and fibrinolysis-related parameters in predicting postoperative venous thrombosis in patients with breast cancer
}

\author{
Mengyu Pang ${ }^{1}$, Fenglian Zhao ${ }^{1}$, Pengyue Yu ${ }^{1}$, Xiaohua Zhang ${ }^{1}$, Hexin Xiao ${ }^{1}$, Wang Qiang ${ }^{2}$, \\ Hongquan Zhu' ${ }^{1}$, Liyan Zhao ${ }^{1}$ \\ ${ }^{1}$ Department of Laboratory, The Second Hospital of Jilin University, Changchun, China; ${ }^{2}$ Business Department, Sekisui Medical Technology (China) \\ Ltd., Beijing, China \\ Contributions: (I) Conception and design: M Pang, H Zhu, L Zhao; (II) Administrative support: H Zhu, L Zhao; (III) Provision of study materials \\ or patients: H Xiao, W Qiang; (IV) Collection and assembly of data: P Yu, X Zhang; (V) Data analysis and interpretation: M Pang, F Zhao; (VI) \\ Manuscript writing: All authors; (VII) Final approval of manuscript: All authors. \\ Correspondence to: Hongquan Zhu; Liyan Zhao. Department of Laboratory, The Second Hospital of Jilin University, No. 218, Ziqiang Street, \\ Nanguan District, Changchun 130022, China. Email: hongquanzhu1966@126.com;970201043@qq.com.
}

\begin{abstract}
Backgrounda To explore the expression level of coagulation and fibrinolysis-related indexes in the plasma of breast cancer patients after surgery, and explore their predictive value for deep venous thrombosis (DVT).

Methods: From May 2016 to May 2019, 63 patients with lower extremity DVT after radical mastectomy in our hospital were selected as the thrombus group, and 69 patients without venous thrombosis after radical mastectomy were selected as the control group. The levels of D-dimer (D-D) and fibrinolytic product (FDP) were measured by latex enhanced immunoturbidimetry, Fibrinogen (FIB) levels were measured using the von Clauss method, thrombin antithrombin complex (TAT) and thrombomodulin (TM) levels were measured by enzyme-linked immunosorbent assay (ELISA), and the evaluation value of coagulation markers on tumor thrombosis was analyzed by receiver operating characteristic curve (ROC) curve analysis.

Results: There were significant differences in blood pressure, platelet count (PLT) level, diabetes history, and tumor metastasis between the two groups $(\mathrm{P}<0.05)$. The levels of PT, D-D, FDP, TAT, and TM in the thrombus group were significantly higher than those in control group $(\mathrm{P}<0.05)$. The area under the curve (AUC) of D-D, FDP, and TAT were $0.790,0.881$, and 0.672 , respectively and there was a marked difference among the indexes $(\mathrm{P}<0.05)$. The AUC of FDP was the largest, and the sensitivity and diagnostic value of FDP were the highest.

Conclusions: The plasma levels of FDP, D-D, TAT, and TM in breast cancer patients with DVT after radical mastectomy were significantly increased, which is related to imbalanced coagulation and fibrinolysis functioning in patients. FDP had the highest predictive value for DVT after radical mastectomy.
\end{abstract}

Keywords: Coagulation function index; fibrinolysis function index; breast cancer; postoperative; deep venous thrombosis (DVT)

Submitted Jan 28, 2021. Accepted for publication Apr 20, 2021.

doi: $10.21037 /$ gs-21-117

View this article at: http://dx.doi.org/10.21037/gs-21-117

\section{Introduction}

Breast cancer is a common gynecological tumor, which has a high incidence in middle-aged and elderly women and seriously impacts the health and lives of patients (1). At present, surgery is the primary clinical treatment of early and mid-term breast cancer to improve the survival rate of patients (2). However, patients are prone to postoperative complications, such as deep vein thrombosis (DVT), which 
is one of the main causes of death (3). A study has shown that the annual incidence of DVT in cancer patients is much higher than that in healthy people (4). DVT mostly occurs in the lower extremities, especially the lower legs (5). It has been found that the destruction of the homeostatic equilibrium of the coagulation and fibrinolytic systems, caused by vascular endothelial injury and increased inflammatory response, can easily lead to DVT (6). The thrombin antithrombin complex (TAT) is an important coagulation marker, and an increased level of TAT can portend early abnormal coagulation function in the body (7). Thrombomodulin (TM) levels are abnormally up-regulated when the vascular endothelium is damaged, which helps to assess the degree of vascular injury (8). Fibrinogen degradation product (FDP) and D-dimer (D-D) are both functional markers of the fibrinolytic system, and their levels are significantly increased when fibrinolytic activity is enhanced in patients, which can indicate blood coagulation system activity $(9,10)$. These are important markers in the process of venous thrombosis, which can be significantly increased during thrombosis. Therefore, in this study, we evaluated the diagnostic and prognostic value of changes in the coagulation and fibrinolytic function index for detecting DVT in the lower extremities of patients with breast cancer after radical mastectomy.

We present the following article in accordance with the STROBE reporting checklist (available at http://dx.doi. org/10.21037/gs-21-117).

\section{Methods}

\section{Clinical data}

This was a prospective observational study. From May 2016 to May 2019, 63 patients with lower extremity DVT after radical mastectomy in our hospital were selected as the thrombus group, and 69 patients without venous thrombosis after radical mastectomy were selected as the control group. The inclusion criteria were as follows: (I) patients diagnosed with breast cancer according to pathological tissue examination, imaging examination results, and breast cancer-related diagnostic criteria (11); (II) patients that required modified radical mastectomy treatment for unilateral breasts; (III) breast cancer patients with lower extremity DVT according to the diagnostic criteria in the Guidelines for the Diagnosis and Treatment of DVT (12) issued by the Vascular Surgery Group of the Surgery Branch of the Chinese Medical Association; and (IV) patients with complete clinical data. The exclusion criteria were as follows: (I) patients with recurrent breast cancer; (II) patients treated with anticoagulant drugs; (III) patients with severe deficiencies in liver or kidney function; and (IV) patients with mental illness. This study was approved by the Ethics Committee of the Ethics Committee of The Second Hospital of Jilin University (No. 2019087). All procedures performed in this study involving human participants were in accordance with the Declaration of Helsinki (as revised in 2013). Informed consent was taken from all the patients.

\section{Main instruments and reagents}

Coagulation function analyzer (model: CP-2000, Sysmex, Japan), D-D detection kit [article number: 483820 , Sekisui Medical Technology (China) Ltd., Beijing, China], fibrinogen (FIB) detection kit [article number: GMS10367, Sekisui Medical Technology (China) Ltd., Beijing, China], FDP detection kit [article number: 483790, Sekisui Medical Technology (China) Ltd., Beijing, China], TAT ELISA kit (article number: ab108907, Abcam, USA), TM ELISA kit (article number: orb154577, Biorbyt, UK).

\section{General information collection}

The patients' age, body mass index (BMI), systolic blood pressure (SBP), diastolic blood pressure (DBP), smoking history, drinking history, tumor metastasis condition, tumor subtype, diabetes history, as well as the levels of platelet count (PLT), triglycerides (TG), total cholesterol (TC), high-density lipoprotein (HDL), and low-density lipoprotein (LDL) were recorded.

\section{Sample collection and detection of coagulation and fibrinolysis-related indexes}

Prior to a patient's postoperative prophylactic treatment with low-molecular heparin anticoagulants, $4 \mathrm{~mL}$ of fasting blood in the morning was collected using citrate anticoagulant vacuum blood collection tubes, and the blood was tested for routine coagulation indexes after centrifugation. Prothrombin time (PT), activated partial thromboplastin time (APTT), and thrombin time (TT) were detected using a coagulation function analyzer.

D-D and FDP levels were detected by latex-enhanced immunoturbidimetry assay. Tris buffer and antibodies were mixed into the kit and placed into an automatic coagulation analyzer to determine the calibration data. The patients' plasma samples were then placed into a coagulation analyzer 


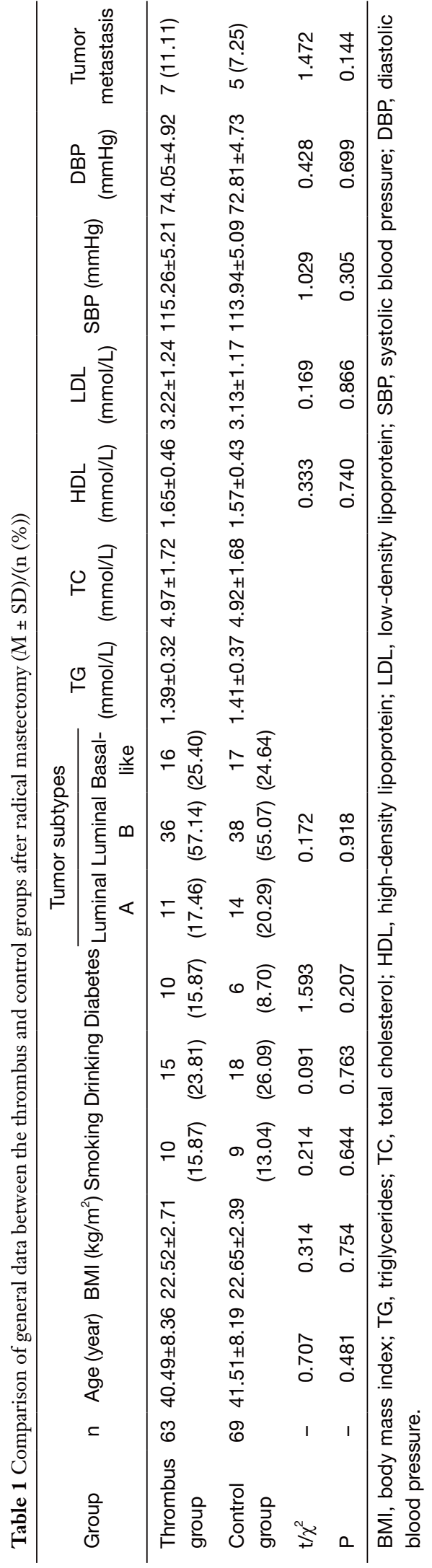

for detecting D-D and FDP levels.

The von Clauss method was used to detect the FIB level. The plasma was calibrated using the calibration reagent in the kit and the automatic coagulation analyzer, and was subsequently placed in the coagulation analyzer together with bovine thrombin and calibrated plasma.

Platelet-poor plasma was taken and the levels of coagulation and fibrinolytic markers, TAT and TM, were measured using a chemiluminescent ELISA kit according to the manufacturer's instructions.

\section{Statistical analysis}

Statistical analysis was performed using SPSS 22.0 (International Business Machines Corporation, Armonk, State of New York, USA). Count data was expressed as "case (\%)", and the chi-square $\left(\chi^{2}\right)$ test was performed. Measurement data was expressed as the mean standard deviation $(M \pm S D)$, and the $t$-test was performed between the groups. The receiver operating characteristic curve (ROC) curve was used to analyze the evaluation value of coagulation markers for tumor thrombosis, including sensitivity, specificity, Youden index, and the best diagnostic value (the ROC curve point closest to the upper left corner). The area under the ROC curve (AUC) was compared using the $Z$ test. $\mathrm{P}<0.05$ indicated a statistically significant difference.

\section{Results}

\section{Comparison of general data between the thrombus and control groups after radical mastectomy}

Patients in the thrombus group were aged between 26 and 51 years, with an average of age of $40.49 \pm 8.36$ years. Patients in the control group were aged between 27 and 51 years, with an average of age of $41.51 \pm 8.19$ years. Among the enrolled patients, 41 cases were in stage $I$ and 91 cases were in stage II. There were no statistically significant differences between the groups in terms of age, weight, smoking history, drinking history, history of diabetes mellitus, proportion of patients with tumor metastases, blood pressure, tumor subtype, or in the levels of TG, TC, HDL, and LDL $(\mathrm{P}>0.05)$ (Table 1).

\section{Comparison of plasma routine coagulation index levels between the thrombus and control groups after radical mastectomy}

As shown in Table 2 and Figure 1, in the thrombus group 
Table 2 Comparison of plasma routine coagulation index levels between the thrombus and control groups after radical mastectomy $(\mathrm{M} \pm \mathrm{SD})$

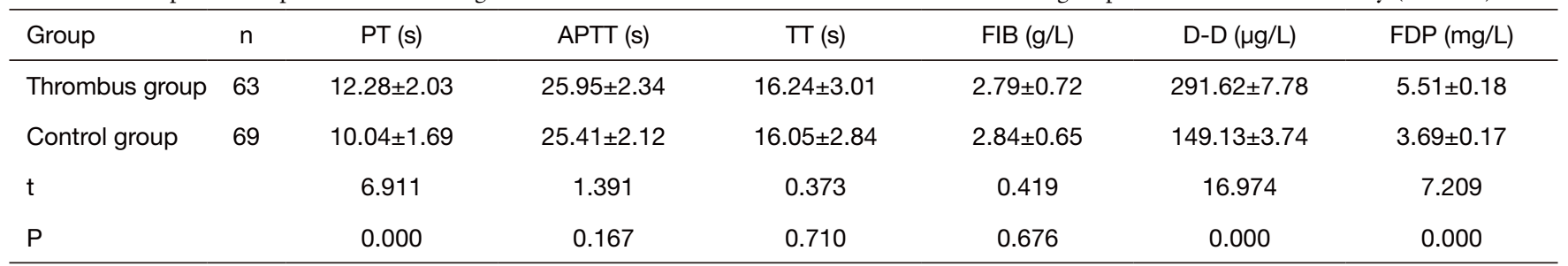

PT, prothrombin time; APTT, activated partial thromboplastin time; TT, thrombin time; FIB, fibrinogen; D-D, D-dimer; FDP, fibrinolytic product.

A

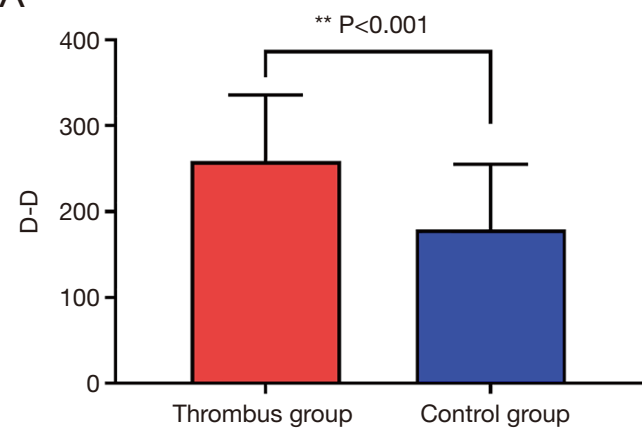

B

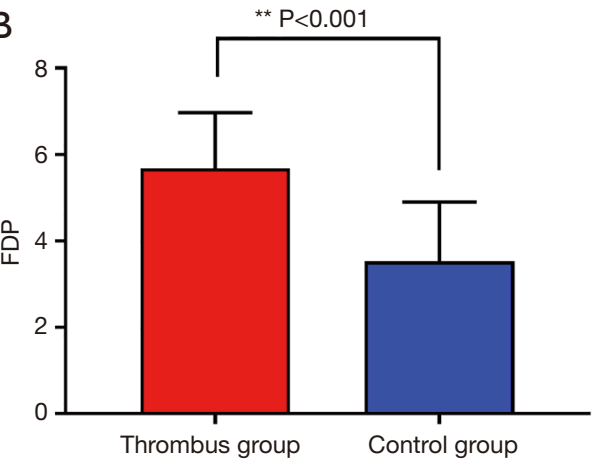

Figure 1 Comparison of D-D and FDP levels between the thrombus and control groups. (A) Comparison of D-D level between the thrombus and control groups; (B) comparison of FDP level between the thrombus and control groups. D-D, D-dimer; FDP, fibrinolytic product. ${ }^{* *}, \mathrm{P}<0.01$.

Table 3 Comparison of TAT and TM levels in platelet-poor plasma after radical mastectomy $(\mathrm{M} \pm \mathrm{SD})$

\begin{tabular}{lccc}
\hline Group & $\mathrm{n}$ & $\mathrm{TAT}(\mu \mathrm{g} / \mathrm{L})$ & $\mathrm{TM}(\mu \mathrm{g} / \mathrm{L})$ \\
\hline Thrombus group & 63 & $5.97 \pm 0.22$ & $2.23 \pm 0.18$ \\
Control group & 69 & $5.04 \pm 0.21$ & $1.62 \pm 0.14$ \\
$\mathrm{t}$ & & 3.132 & 2.684 \\
$\mathrm{P}$ & & 0.002 & 0.008 \\
\hline
\end{tabular}

TAT, thrombin antithrombin complex; TM, thrombomodulin.

with coagulopathy, the levels of PT, D-D, and FDP were significantly higher than those of the tumor control group, and the difference was statistically significant $(\mathrm{P}<0.05)$. There was no statistically significant difference in APTT, TT, and FIB levels between the two groups $(\mathrm{P}>0.05)$.

Comparison of TAT and TM levels in platelet-poor plasma between the thrombus and control groups after radical mastectomy

The levels of TAT and TM in the thrombus group were markedly higher than those in the control group, and the difference was statistically significant (Table 3 and Figure 2).

\section{Evaluation efficiency of coagulation markers in the diagnosis of tumor thrombosis}

As shown in Figure 3 and Table 4, ROC curve analysis showed that the AUCs of D-D, FDP, and TAT were 0.790 , 0.881 , and 0.672 , respectively, and the differences among the indexes were statistically significant (D-D vs. FDP, FDP vs. TAT, D-D vs. TAT; comparison $Z$ value $=2.067,3.802$, 1.967 , respectively; all $\mathrm{P}<0.05)$. The AUC of TAT was 0.625; compared with the AUC of TM, the difference was not statistically significant $(\mathrm{P}>0.05)$. The AUC of FDP was the largest, and the Youden index was the highest, with the highest diagnostic value.

\section{Discussion}

DVT refers to a venous return disorder caused by the abnormal clotting of blood in the deep vein cavity that obstructs the lumen (13). Venous endothelial injury, slow 

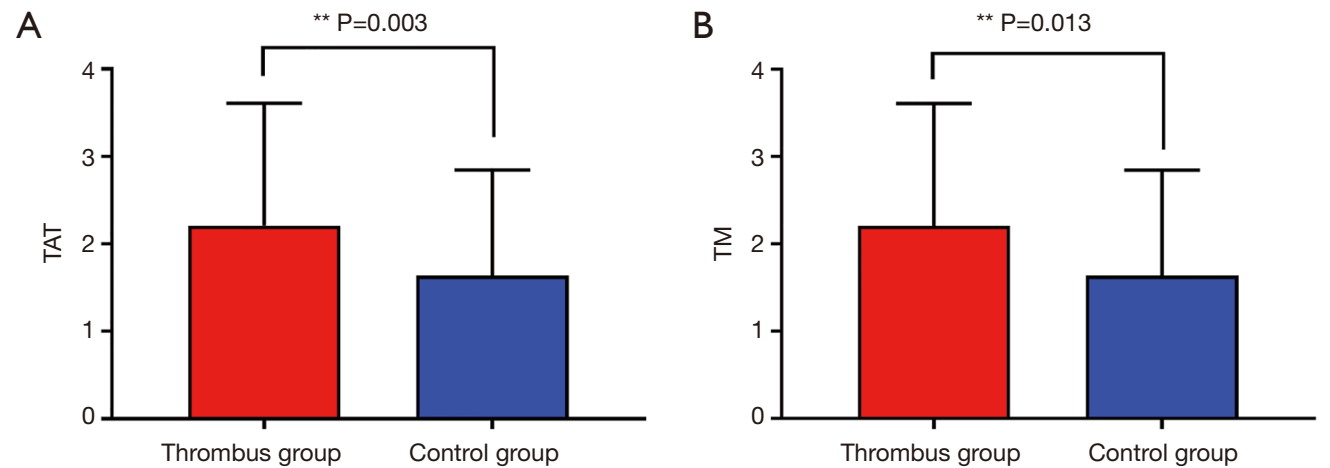

Figure 2 Comparison of TAT and TM levels between the thrombus and control groups. (A) Comparison of TAT level between the thrombus and control groups; (B) comparison of TM level between the thrombus and control groups. * ${ }^{*}<0.05 ;{ }^{* *}, \mathrm{P}<0.01$. TAT, thrombin antithrombin complex; TM, thrombomodulin.

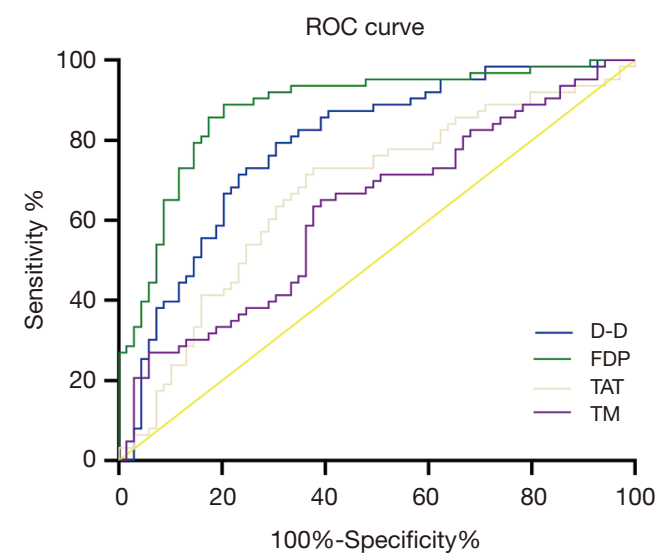

Figure 3 ROC curve of the evaluation efficacy of coagulation markers in the diagnosis of tumor thrombosis.

blood flow, and abnormal coagulation status after radical mastectomy are the main causes of postoperative DVT in patients (14). Mechanical trauma during surgery can cause vascular endothelial injury, which is also one of the risk factors for postoperative DVT in breast cancer patients (15). Tumor cells themselves can also target related factors to activate prothrombin expression in order to regulate the body's coagulation system, or target fibrinolysis-related proteins and promote the increase of fibrinase levels in order to regulate the fibrinolytic system, thereby leading to an increase in blood viscosity and promotion of DVT (16).

Under normal conditions, the coagulation and fibrinolytic systems in the human body maintain a dynamic balance. However, the body's stress response and damage to vascular endothelial cells during surgery can raise the level of fibrin regulatory factors, resulting in the blood continuously being in a hypercoagulable state, which is the basis of thrombosis (17). TAT is the product of the interaction between thrombin and antithrombin, which can accurately reflect the level of thrombin in the body (18). A study has shown that plasma TAT levels in middleaged and elderly patients are significantly increased after traumatic artificial hip replacement, which can be used as an early diagnostic marker for patients who experience DVT after surgery (19). Another study reported that the high expression of plasma TAT in patients with malignant tumors could be used to diagnose thrombosis by reflecting the abnormal enhancement of coagulation and fibrinolytic activity in patients (20).

As a thrombin-regulating protein, TM can also predict the level and changes in the body's coagulation function (21). Increased plasma TM levels in patients after hysterectomy can indicate exacerbation of vascular endothelial damage and the hypercoagulable state of the body (22). The plasma TM level of patients after bone trauma is elevated, which is related to the occurrence of thrombosis after surgery, and can be used to predict venous thrombosis (23). The results of this study showed that the levels of TAT and TM in the thrombus group were significantly higher than those of the control group, suggesting that TAT and TM may portend an increased risk of lower extremity DVT by reflecting the enhanced coagulation function and hypercoagulable state of breast cancer patients after radical mastectomy.

FDP is a degradation product produced by FIB under the action of plasmin, and increased levels of FDP can reflect increased fibrinolytic activity in the body (24). Increased FDP levels after laparoscopic surgery can predict the 
Table 4 Evaluation efficiency of blood coagulation indexes in the diagnosis of tumor thrombosis

\begin{tabular}{lcccccc}
\hline Testing index & Cut off value & AUC & Sensitivity (\%) & Specificity (\%) & Youden index & $95 \% \mathrm{Cl}$ \\
\hline D-D & $198.50 \mu \mathrm{g} / \mathrm{L}$ & 0.790 & 79.37 & 69.57 & 0.489 & $0.710-0.856$ \\
FDP & $4.95 \mathrm{mg} / \mathrm{L}$ & 0.881 & 88.89 & 79.71 & 0.686 & $0.813-0.931$ \\
TAT & $6.19 \mu \mathrm{g} / \mathrm{L}$ & 0.672 & 73.02 & 62.32 & 0.353 & $0.585-0.751$ \\
TM & $2.26 \mu \mathrm{g} / \mathrm{L}$ & 0.625 & 65.08 & 60.87 & 0.625 & $0.537-0.708$ \\
\hline
\end{tabular}

AUC, area under the ROC curve; D-D, D-dimer; FDP, fibrinolytic product; TAT, thrombin antit.

occurrence of venous thrombus embolism in patients (25). The increased expression of FDP in the plasma of patients with venous catheterization after lung cancer surgery is closely related to the occurrence of thrombosis, and the AUC of FDP for the diagnosis of thrombosis occurrence is $0.816(26)$.

$\mathrm{D}-\mathrm{D}$ is a specific fibrinolysis marker, and increased levels of D-D may indicate increased fibrinolysis activity in the body. The levels of plasma D-D in patients with acute lower limb DVT after total knee replacement is significantly higher than that of patients without acute lower limb DVT, which has a certain screening value for DVT (27). Up-regulation of D-D levels can be used to predict postoperative occurrence of portal vein thrombosis in patients with portal hypertension (28).

The results of this study showed that the levels of PT, $\mathrm{D}-\mathrm{D}$, and FDP in the thrombus group with coagulopathy were significantly higher than those in the tumor control group, suggesting that the increased levels of D-D and FDP could predict the occurrence of lower extremity DVT in patients with breast cancer after radical mastectomy. This process may be related to intraoperative vascular endothelial injury, which stimulates the expression of coagulationrelated proteins and leads to an imbalance between the coagulation and the fibrinolytic systems. Therefore, these biomarkers may play an important role in the diagnosis of DVT in patients after radical mastectomy.

In this study, ROC curve analysis showed that the AUCs of FDP, D-D, and TAT in predicting lower extremity DVT in patients with breast cancer after radical mastectomy were $0.881,0.790$, and 0.672 , respectively, and there were significant differences among these indexes. The predictive sensitivities of FDP, D-D, TAT, and TM were $88.89 \%$, $79.37 \%, 73.02 \%$, and $65.08 \%$, respectively, indicating that the predictive value of plasma FDP for breast cancer patients with lower extremity DVT after undergoing radical mastectomy was higher than the other indicators.
Clinically, more attention should be paid to the changes in FDP levels of patients with breast cancer after radical surgery in order to more accurately assess the occurrence of DVT of the lower extremities. However, the AUC of TM was 0.625 , which was not significantly different from that of TAT, indicating that both of these indicators have similar predictive value for the occurrence of lower extremity DVT after surgery.

In conclusion, plasma FDP, D-D, TAT, and TM levels were significantly increased in breast cancer patients who developed lower-extremity DVT after radical mastectomy, which was related to imbalanced coagulation and fibrinolytic functioning in patients. Moreover, FDP had the highest predictive value for occurrence of lower extremity DVT after radical mastectomy. However, the mechanisms of the various indexes involved in the occurrence of DVT need to be further verified and studied.

\section{Acknowledgments}

Funding: None.

\section{Footnote}

Reporting Checklist: The authors have completed the STROBE reporting checklist. Available at http://dx.doi. org/10.21037/gs-21-117

Data Sharing Statement: Available at http://dx.doi. org/10.21037/gs-21-117

Conflicts of Interest: All authors have completed the ICMJE uniform disclosure form (available at http://dx.doi. org/10.21037/gs-21-117). The authors have no conflicts of interest to declare.

Ethical Statement: The authors are accountable for all 
aspects of the work in ensuring that questions related to the accuracy or integrity of any part of the work are appropriately investigated and resolved. This study was approved by the Ethics Committee of The Second Hospital of Jilin University (No. 2019087). All procedures performed in this study involving human participants were in accordance with the Declaration of Helsinki (as revised in 2013). Informed consent was taken from all the patients.

Open Access Statement: This is an Open Access article distributed in accordance with the Creative Commons Attribution-NonCommercial-NoDerivs 4.0 International License (CC BY-NC-ND 4.0), which permits the noncommercial replication and distribution of the article with the strict proviso that no changes or edits are made and the original work is properly cited (including links to both the formal publication through the relevant DOI and the license). See: https://creativecommons.org/licenses/by-nc-nd/4.0/.

\section{References}

1. Parada H Jr, Gammon MD, Ettore HL, et al. Urinary concentrations of environmental phenols and their associations with breast cancer incidence and mortality following breast cancer. Environ Int 2019;130:104890.

2. Zou L, Liu F, Shen $P$, et al. The incidence and risk factors of related lymphedema for breast cancer survivors postoperation: a 2-year follow-up prospective cohort study. Breast Cancer 2018;25:309-14.

3. Zhang C, Xiong X, Deng S, et al. Clinical Study of Aspirin in the Prevention of Thrombosis in Breast Cancer Patients with Postoperative Chemotherapy after PICC Insertion. Yangtze Med 2018;2:255-61.

4. Tuzovic M, Herrmann J, Iliescu C, et al. Arterial thrombosis in patients with cancer. Curr Treat Options Cardiovasc Med 2018;20:40.

5. Faria C, Antunes H, Pontes T, et al. Deep venous thrombosis of lower limbs in adolescents:a study in a tertiary hospital. Int J Adolesc Med Health 2019;1:1-7.

6. Mukai M, Oka T. Mechanism and management of cancerassociated thrombosis. J Cardiol 2018;72:89-93.

7. Ebara S, Marumo M, Mukai J, et al. Relationships of oxidized HDL with blood coagulation and fibrinolysis in patients with type 2 diabetes mellitus. J Thromb Thrombolysis 2018;45:200-5.

8. Deng HF, Wang S, Li L, et al. Puerarin prevents vascular endothelial injury through suppression of NF- B activation in LPS-challenged human umbilical vein endothelial cells.
Biomed Pharmacother 2018;104:261-7.

9. Lostak J, Gallo J, Slavik L, et al. Monitoring of fibrinolytic system activity with plasminogen, D-dimers and FDP in primary total knee arthroplasty (TKA) after topical, intravenous or combined administration of tranexamic acid. Biomed Pap Med Fac Univ Palacky Olomouc Czech Repub 2020;164:168-76.

10. Görlinger K. Biomarkers versus viscoelastic testing for the detection of fibrinolysis. ANZ J Surg 2020;90:411-2.

11. Sinn HP, Helmchen B, Wittekind CH. TNM classification of breast cancer:changes and comments on the 7 th edition. Der Pathologe 2010;31:361-6.

12. Chinese society for cardiovascular surgery. Guidelines for the diagnosis and treatment of deep vein thrombosis. Chinese Journal of General Surgery 2008;23:235-8.

13. Needleman L, Cronan JJ, Lilly MP, et al. Ultrasound for lower extremity deep venous thrombosis: multidisciplinary recommendations from the Society of Radiologists in Ultrasound Consensus Conference. Circulation 2018;137:1505-15.

14. Cui LN, Li N, Fu S, et al. Combination of preoperative D-dimer and mean platelet volume predicts postoperative deep venous thrombosis in breast cancer patients. CANCER BIOMARK 2018;21:909-13.

15. Poredos P, Jezovnik MK. Endothelial dysfunction and venous thrombosis. Angiology 2018;69:564-7.

16. Hisada Y, Mackman N. Cancer cell-derived tissue factorpositive extracellular vesicles: biomarkers of thrombosis and survival. Curr Opin Hematol 2019;26:349-56.

17. Ogbechi J, Ruf MT, Hall BS, et al. Mycolactonedependent depletion of endothelial cell thrombomodulin is strongly associated with fibrin deposition in Buruli ulcer lesions. PLoS Pathog 2015;11:e1005011.

18. Bai F, Feng S, Xu C, et al. Transurethral resection versus holmium laser enucleation of the prostate: A prospective randomized trial comparing perioperative thrombin generation and fibrinolysis. Medicine (Baltimore) 2019;98:e15223.

19. Song YC, Deng YJ, Liu ZF, et al. Perioperative blood management combined with Bazhen Tang improves hemoglobin and hypercoagulability in senile patients after joint replacement. Chin J Tissue Engineer Res 2016;20:1-8.

20. Yang C, Sun W, Cui W, et al. Procoagulant role of neutrophil extracellular traps in patients with gastric cancer. Int J Clin Exp Pathol 2015;8:14075.

21. Daly C, Qian X, Castanaro C, et al. Angiopoietins bind thrombomodulin and inhibit its function as a thrombin 
cofactor. Sci Rep 2018;8:505.

22. Kobayashi T, Kajiki M, Nihashi K, et al. Surveillance of the safety and efficacy of recombinant human soluble thrombomodulin in patients with obstetrical disseminated intravascular coagulation. Thromb Res 2017;159:109-15.

23. Cheng TL, Lai CH, Shieh SJ, et al. Myeloid thrombomodulin lectin-like domain inhibits osteoclastogenesis and inflammatory bone loss. Sci Rep 2016;6:28340.

24. Bai Y, Shi M, Yang X, et al. The value of FDP/FIB and D-dimer/FIB ratios in predicting high-risk APL-related thrombosis. Leuk Res 2019;79:34-7.

25. Yang C, Zhu L. Coagulation and deep vein flow changes following laparoscopic total extraperitoneal inguinal hernia repair:a single-center, prospective cohort study. Surg Endosc 2019;33:4057-65.

Cite this article as: Pang M, Zhao F, Yu P, Zhang X, Xiao H, Qiang W, Zhu H, Zhao L. The significance of coagulation and fibrinolysis-related parameters in predicting postoperative venous thrombosis in patients with breast cancer. Gland Surg 2021;10(4):1439-1446. doi: 10.21037/gs-21-117
26. Tian B, Song C, Li H, et al. The significance of perioperative coagulation and fibrinolysis related parameters after lung surgery for predicting venous thromboembolism: a prospective, single center study. J Thorac Dis 2018;10:1-10.

27. Mitani G, Takagaki T, Hamahashi K, et al. Associations between venous thromboembolism onset, D-dimer, and soluble fibrin monomer complex after total knee arthroplasty. J Orthop Surg Res 2015;10:172.

28. Malaguarnera M, Latteri S, Bertino G, et al. D-dimer plasmatic levels as a marker for diagnosis and prognosis of hepatocellular carcinoma patients with portal vein thrombosis. Clin Exp Gastroenterol 2018;11:373-80.

(English Language Editor: A. Kassem) 